\title{
Sex Differences in Social Interaction in Rats: Role of the Immediate-Early Gene zif268
}

\author{
Ashley Stack ${ }^{1,2}$, Nicole Carrier ${ }^{1,2}$, David Dietz', Fiona Hollis', Jamie Sorenson' and Mohamed Kabbaj*,' \\ 'Department of Biomedical Sciences, Program in Neurosciences, College of Medicine, Florida State University, Tallahassee, FL, USA
}

\begin{abstract}
Given both the high prevalence of anxiety disorders in women and the fact that little is known about the mechanisms of gender differences in anxiety, our primary aim in this study was to investigate the neurobiological mechanisms underlying sex differences in social anxiety-like behavior in rats. Through the use of zif268 antisense oligodeoxynucleotides (zif ASO), we induced a temporary downregulation of zif268 expression in the medial prefrontal cortex of male and female rats and found that zif268 ASO male rats show more social anxiety-like behaviors when compared with control male rats in the social interaction test. In fact, zif268 ASO males displayed social anxiety-like behaviors, which were similar to control females, thus downregulation of zif268 expression in the mPFC of male rats eliminated sex differences previously found in the social anxiety-like behavior tests. Interestingly, zif268 ASO in female rats had no effect on their social interaction. Our novel findings have led us to ascertain that sexually dimorphic zif268 expression in the mPFC is a key molecular factor in mediating sex-specific anxiety-like behavior in the social interaction test. Neuropsychopharmacology (2010) 35, 570-580; doi:10.1038/npp.2009.163; published online 21 October 2009
\end{abstract}

Keywords: anxiety; sex differences; zif268; prefrontal cortex

\section{INTRODUCTION}

There are largely documented sex differences in anxiety and mood disorders, where women are more than twice as likely as men to suffer from depression and anxiety disorders (Gater et al, 1998; Kessler et al, 1994). In addition to these sex differences found in general anxiety disorders, many studies have also found that women are more likely to suffer from social anxiety disorders than men (Kessler et al, 1994; Schneier et al, 1992). Because only women have an increased rate of incidence for anxiety and other mood disorders during the period after puberty and before menopause (Kessler et al, 1994), estrogen has been widely studied as being an important factor in the etiology and maintenance of anxiety in women of reproductive age. Given the high proportion of females that suffer from anxiety disorders, it is surprising that many animal studies dealing with anxiety have been performed using only males. Overall, it seems that there may be profound sex differences in anxiety-like behavior and in the way that males and females react to stressful or threatening stimuli, thus resulting in the need for more studies delving into the

*Correspondence: Dr Mohamed Kabbaj, Department of Biomedical Sciences and Neurosciences, Florida State University, College of Medicine, III 5 W Call Street, Tallahassee, Florida, 32306, USA,

Tel: + 850644 4930, E-mail: mohamed.kabbaj@med.fsu.edu

${ }^{2}$ Ashley Stack and Nicole Carrier are co-first authors

Received 12 June 2009; revised 14 August 2009; accepted I September 2009 mechanisms, which mediate sex differences in anxiety responses.

One of the aims of this study was to determine if there were any sex differences in anxiety-like behaviors in rats, especially in the context of environment-dependent social behavior. To this end, we used the social interaction (SI) test, which has been shown to have a high degree of etiological validity. Sex differences have been previously reported in the SI test, in that adult male rats spent more time engaging in SI (and thus, were less socially anxious) than their female counterparts (Johnston and File, 1991). However, the actions of ovarian hormones were not taken into account or controlled for in this study. Given the little research conducted on sex differences in the SI test, we wanted to elaborate on this study and determine whether the ovarian hormone $17 \beta$-estradiol would increase or decrease female SI relative to their male counterparts.

A second aim of our study was to characterize mRNA expression patterns of the immediate-early gene zif268 (also known as egr-1, krox24, NGFI-A, and zenk) throughout the brain in both male and female rats to determine if there was sexually dimorphic expression of zif268 following SI and also in basal stress-free conditions. Zif268, like other inducible transcription factors, acts as a transcriptional switch that can enhance or inhibit transcription of several other genes, such as those involved in synaptic plasticity (Goelet et al, 1986; Lisman, 1999). Hence, zif268 expression can lead to long-lasting synaptic changes in the brain by activating specific signaling pathways by means of its inducible transcription and subsequent protein synthesis 
(Davis and Squire, 1984). It is this mechanism that is the basis for many recent studies implicating zif268 in mediating certain types of behavior, such as learning and memory formation (Cole et al, 1989), fear conditioning (Malkani et al, 2004; Ressler et al, 2002), and drug addiction (Valjent et al, 2006). Altogether, there is rapidly accumulating evidence suggesting that zif268 expression in specific brain areas is involved in the mechanisms underlying certain behaviors of interest, especially those behaviors, which have a strong learning component.

The final aim of this study was to examine the functional significance of the sexually dimorphic zif268 mRNA and protein expression in mPFC (dorsal and ventral mPFC). Indeed, male rats had both higher zif268 expression in the mPFC and higher SI than their female counterparts. We therefore decided to investigate the effects of downregulation of zif268 expression in the mPFC on SI test by infusing zif268 ASO into the mPFC of male and female rats before SI testing and comparing their social interaction to that of control males and females infused with zif268 missense oligodeoxynucleotides (MSO). In other words, we wanted to know if a decrease in zif268 expression in the mPFC of male rats would serve to decrease their SI and make their behavior similar to that of females, in essence abolishing sex differences previously observed in social anxiety-like behaviors in the SI test.

\section{MATERIALS AND METHODS}

\section{Animals}

Adult Sprague-Dawley (SD) rats (males: 250-270 g; females: 180-200 g) were used in all studies. Ovariectomized (OVX) females and SHAM males as well as intact males and females were used. OVX and Sham rats were purchased from Charles River and were treated with E2/Oil 10 days after the surgeries. Rats were housed in same-sex pairs in $43 \times 21.5 \times 25.5 \mathrm{~cm}$ Plexiglas cages and kept on a $12: 12 \mathrm{~h}$ light/dark cycle (lights on at $0700 \mathrm{~h}$ ). Food and water were available ad libitum. All animal protocols were in accordance with the NIH Guide for Care and Use of Laboratory Animals and were approved by our Institutional Animal Care and Use Committee.

Experiment 1: Does E2 Affect Sex Differences In Social Interaction? Are There Sex Differences in Zif268 mRNA Expression in the Brain Following SI?

In this experiment, we used OVX female rats and SHAM male rats. Female rats were assigned to four treatment groups: (1) $10 \mu \mathrm{g} 17 \beta$-estradiol (E2) $n=12$; (2) $5 \mu \mathrm{g}$ E2 $n=12$; (3) $2 \mu \mathrm{g}$ E2 $n=12$; and (4) sesame oil (OIL) $n=12$. Male rats were only given OIL treatment and received no hormone replacement $(n=12)$. All treatments were in the form of one acute subcutaneous injection, followed $48 \mathrm{~h}$ later by behavioral testing in the SI test, consisting of $10 \mathrm{~min}$ habituation in an open field followed by a $10 \mathrm{~min}$ social interaction with an unfamiliar conspecific rat (OVX females and SHAM males). Rats were killed immediately after the $20 \mathrm{~min}$ of behavioral testing in the SI test. All rats were decapitated and brains were rapidly removed, snap frozen in 2-methylbutane chilled to $-35^{\circ} \mathrm{C}$, and stored at $-80^{\circ} \mathrm{C}$ until further processing for zif268 in situ hybridization.
The time of killing ( $48 \mathrm{~h}$ after E2/oil injections) was chosen based on a previous publication showing the antianxiety effects of E2 when injected $48 \mathrm{~h}$ before testing (Walf and Frye, 2005).

\section{Experiment 2: Does Estrous Cycle Affect Social Interaction?}

In this experiment, 16 intact male rats and 30 intact female rats (14 in proestrous and 16 in diestrous) were used. Stage of the estrous cycle (diestrous 1, diestrous 2, proestrous, or estrous) was determined by examining the appearance and abundance of cells within vaginal cytology samples. Diestrous 1 was characterized by leukocytes and clusters of cornified cells, and proestrous was characterized primarily by nucleated epithelial cells. Before running rats in the SI test, they were habituated to the vaginal smears for at least two estrous cycles. On test day, rats were lavaged $2 \mathrm{~h}$ before the SI behavior. Male rats were handled daily for $1 \mathrm{~min}$. The unfamiliar conspecific rats used in this experiment were intact male rats or female rats in their diestrous cycle. These rats were used during only one social interaction test.

Experiment 3: Are There Sex Differences in zif268 mRNA and Protein Expression in the Brain in Basal Stress-Free Conditions?

In this experiment, eight OVX female rats and eight sham male rats were injected with sesame oil and killed $48 \mathrm{~h}$ later in stress-free conditions to examine the basal expression of zif268 mRNA.

Another six intact female rats and seven intact male rats were killed in stress-free conditions to examine the basal expression of zif268 protein in mPFC by western blotting.

\section{Experiment 4: What is the Functional Significance of Sex} Differences in zif268 Expression in the mPFC?

In this experiment, intact male and female rats were used. After handling the rats for 1 week, rats were implanted bilaterally with cannulae aimed at the prelimbic medial prefrontal cortex (PL mPFC; coordinates for males: AP + $2.7 \mathrm{~mm}$; lateral: $\pm 1 \mathrm{~mm}$; DV: $-3 \mathrm{~mm}$; PL mPFC coordinates for females: AP $+3 \mathrm{~mm}$; lateral: $\pm 1 \mathrm{~mm}$; DV: $-3 \mathrm{~mm}$ ). One to two weeks following surgery, rats were connected to a syringe containing either zif268 antisense-ODN (ASO; $0.5 \mathrm{nmol} / \mu \mathrm{l}, 1 \mu \mathrm{l}$ per side dissolved in $\mathrm{PBS}$ at $\mathrm{pH}$ 7.4) or zif268 missense ODN (MSO; $0.5 \mathrm{nmol} / \mu \mathrm{l}, 1 \mu \mathrm{l}$ per side dissolved in $\mathrm{PBS}$ at $\mathrm{pH}$ 7.4). The ASO/MSO solutions were infused into the mPFC over a period of $2 \mathrm{~min}$ in stress-free conditions as described in our previous work (Kabbaj et al, 2000). Seventy minutes after infusion, rats were tested for $10 \mathrm{~min}$ in the open field (locomotor activity and time spent in the center of the field were assessed) and for $10 \mathrm{~min}$ in SI test. This time course was chosen due to previous findings showing that the greatest efficiency for reducing zif268 protein levels was between 1 and $2 \mathrm{~h}$ after zif268 antisense-ODN infusion (Hebb and Robertson, 1997). The injection sites were verified histologically in $35 \mu \mathrm{m}$ Cresyl Violet-stained sections. Out of the 40 rats that received surgery, two rats had improper cannulae placements and 
four lost one of the two implanted cannulae. These six rats (three males and three females) were not used for data analysis.

Before running experiment 4, we have examined the efficiency of our ASO/MSO treatments in eight intact male rats. Rats were injected with either ASO $(n=4)$ or MSO $(n=4)$ in their PL mPFC and then were run in the SI test 70 min later, after which they were killed. Their brains were extracted and stored at $-80^{\circ} \mathrm{C}$ until western blot processing. Also, to ensure proper infusion of zif268 within the mPFC, a biotinylated-zif268 missense oligodeoxynucleotides (zif268 $\mathrm{B}-\mathrm{MSO})$ were infused into the PL $\mathrm{mPFC}$ of four male rats. Their brains were collected, sectioned and used for fluorescence immunolabeling against biotin.

\section{Social Interaction Test}

During the first $10 \mathrm{~min}$ of videotaped testing, the resident rat was placed in a dimly lit open field arena (10-30 lux) and allowed to habituate to its surroundings. Locomotor activity was assessed by partitioning the open field square arena $(90 \times 90 \mathrm{~cm})$ into a $6 \times 6$ square grid and quantifying the total number of grid crosses made by each animal during the first $10 \mathrm{~min}$ of testing. For experiment 4, time spent in the center of the open field was also assessed as a measure of non-social anxiety-like behaviors. After this initial $10 \mathrm{~min}$ in the open field, an unfamiliar conspecific (intruder) rat, that has the same age and weight as the resident rat, was placed in the arena and both rats were allowed to interact for the remaining $10 \mathrm{~min}$ of testing. These unfamiliar conspecific rats were used only once. During quantification of the SI test, the total time spent by the resident rat engaging in active social interaction (ie, sniffing, following, or grooming) with the intruder rat was measured. Because one rat's behavior influences and depends on the other's behavior (see (File and Seth, 2003)), we used only one score (that of the treated resident rat) for each pair.

\section{Tissue Collection and Vaginal Smear}

Tissue collection was performed during the first $4 \mathrm{~h}$ of the light cycle, when levels of circulating corticosterone are at their baseline. Rats were either killed in basal non-stress conditions or immediately after the $20 \mathrm{~min}$ of behavioral testing in the SI test. All rats were decapitated and brains were rapidly removed, snap frozen in 2-methylbutane chilled to $-35^{\circ} \mathrm{C}$, and stored at $-80^{\circ} \mathrm{C}$ until further processing. In the first experiment, a vaginal smear was performed, after rats were killed, to determine the efficacy of E2 treatments on the vaginal tissue. OVX rats treated with oil showed only a small number of leukocytes. OVX rats treated with E2 (2, 5, and 10 UG) showed large clumps of round nucleated epithelial cells, the absence of leukocytes, and, occasionally, a few small clusters of cornified cells, suggestive that $\mathrm{E} 2$ treatment at all the doses induced a proestrous-like cycle in these animals.

\section{In Situ Hybridization}

Eight brains per experimental group were used. Each brain was sectioned on a cryostat at $14 \mu \mathrm{m}$, and a series of sections were mounted on poly-L-lysine-coated slides.
Sections were taken at $100-\mu \mathrm{m}$ intervals, except at the level of the hypothalamus, in which sections were collected at $50 \mu \mathrm{m}$. The sections were fixed in $4 \%$ paraformaldehyde for $1 \mathrm{~h}$, followed by three washes in $2 \times$ saline sodium citrate (SSC). The sections were then placed in a solution containing acetic anhydride $(0.25 \%)$ in triethanolamine $(0.1 \mathrm{M}$, $\mathrm{pH} \mathrm{8)} \mathrm{for} 10 \mathrm{~min}$ at room temperature, rinsed in distilled water and dehydrated through graded alcohols $(50,75,85$, 95 and $100 \%)$. After air-drying, the sections were hybridized with a ${ }^{35}$ S-labeled cRNA probe. The rat zif268 cDNA cloned in our lab yielded a 382-nt cRNA probe. The probe was labeled in a reaction mixture consisting of $1 \mu \mathrm{g}$ of linearized plasmid, $5 \times$ transcription buffer (Epicenter Technologies, Madison, WI, USA), $125 \mu \mathrm{Ci}\left[{ }^{35} \mathrm{~S}\right] \mathrm{UTP}, 125 \mu \mathrm{Ci}\left[{ }^{35} \mathrm{~S}\right] \mathrm{CTP}$, $150 \mu \mathrm{M}$ each of ATP, and GTP, $12.5 \mathrm{mM}$ dithiothreitol, $20 \mathrm{U}$ RNase inhibitor, and $6 \mathrm{U}$ polymerase. The reactions were incubated for $90 \mathrm{~min}$ at $37^{\circ} \mathrm{C}$. The probe was then separated from unincorporated nucleotides over Bio-Rad Micro Bio Spin Chromatography Columns (Bio-Rad Hercules, CA, USA). The probe was diluted in hybridization buffer (containing $50 \%$ formamide, $10 \%$ dextran sulfate, $20 \times$ SSC, $50 \mathrm{mM}$ sodium phosphate buffer, $\mathrm{pH} 7.4,50 \times$ Denhardt's solution, $0.1 \mathrm{mg} / \mathrm{ml}$ yeast tRNA and $10 \mathrm{mM}$ dithiothreitol) to yield $10^{6}$ d.p.m./70 $\mu$ l. The sections were coverslipped and placed inside a humidified box overnight at $55^{\circ} \mathrm{C}$. Following hybridization, the coverslips were removed and the sections rinsed and washed twice in $2 \times$ SSC for $5 \mathrm{~min}$ each, then incubated for $1 \mathrm{~h}$ in RNase $(200 \mu \mathrm{g} /$ $\mathrm{ml}$ in Tris buffer containing $0.5 \mathrm{M} \mathrm{NaCl}, \mathrm{pH} 8$ ) at $37^{\circ} \mathrm{C}$. The sections were washed in increasingly stringent solutions of SSC, $2 \times, 1 \times$ and $0.5 \times$, for $5 \mathrm{~min}$ each, followed by incubation for $1 \mathrm{~h}$ in $0.1 \times \mathrm{SSC}$ at $65^{\circ} \mathrm{C}$. After rinsing in distilled water, the sections were dehydrated through graded alcohols, air-dried and exposed to a Kodak XAR film (Eastman Kodak, Rochester, NY, USA) for 4-7 days.

\section{Quantification of the Radioactive Signal}

As a way to standardize optical density measurements, an outline was developed for each brain region based on the shape and size of the region. Using those outlines, optical density measurements were taken for each brain region from the left and right sides of the brain or from rostral/caudal sections spaced by $100 \mu \mathrm{m}$ or $50 \mu \mathrm{m}$. According to the Paxinos and Watson Atlas, the mPFC was sampled from Bregma 3.7 to Bregma 2.2, the striatum was sampled from Bregma 1.7 to Bregma -0.4 and dorsal hippocampus from Bregma -2.12 to Bregma -4.52 . Accordingly, eight sections per brain region per rat were used. Optical density values were corrected for background, multiplied by the area sampled to produce an integrated density measurement, and then averaged to produce one data point for each brain region for each animal. These data points were averaged per group and compared statistically. Optical density measurements were quantified from X-ray film using Automated Imaging Software (AIS; Imaging Research, St Catherine's, ON, Canada).

\section{Western Blotting}

Total proteins extracted from tissue punches of the mPFC (Bregma 3.7 to Bregma 2.2) were prepared using the Tri Reagent protocol (Molecular Research Center, Cincinnati, 
$\mathrm{OH}$, USA). The areas punched included dorsal mPFC (cingulate cortex 1) and ventral mPFC (prelimbic/infralimbic). $10 \mu \mathrm{g}$ of proteins were run on two SDS- $15 \%$ polyacrylamide gels, and then they were transferred to two nitrocellulose membranes. One membrane was incubated with an anti-zif268 antibody (1:1000) (Santa Cruz Biotechnology Cat. No. Sc-110) and the other membrane with anti-total actin (1:1000) (Sigma-Aldrich, Cat. No. A2066) overnight at room temperature. Membranes were then incubated with peroxidase-labeled AffiniPure goat anti-rabbit secondary antibodies (1:10,000) (Jackson ImmunoResearch Laboratories, Westgrove, PA, USA). Labeled proteins were visualized with enhanced chemiluminescence (ECL SuperSignal West Dura substrate; Pierce Biotechnologies, Rockford, IL, USA) and exposed on Fujifilm XAR film (Fuji Film, Tokyo, Japan). Quantification was performed using AIS 6.0 Image software (Imaging Research, St Catherine's, Ontario, Canada). Data are shown as the ratio of quantified amount of zif268 to quantified amount of total actin in each rat's $\mathrm{mPFC}$.

\section{Zif268 Oligodeoxynucletides}

zif268 oligodeoxynucleotides sequence, dose and volume were adapted from a previous study that showed that zif268 antisense oligodeoxynucleotides (ASO) infused into the amygdala of male rats abolished freezing behaviors in those animals (Malkani et al, 2004). Briefly, the ODNs were purchased from Sigma-Genosys. The ODNs were phosphorothiolated on the last nucleotide at each end to protect against nuclease degradation. Zif268 antisense-ODN was 5'-GGTAGTTGTCCATGGTGG-3 (GenBank accession no. NM 012551, bases 348-365). The nonsense-ODN (5-GTTGGAG TCGGTGGTTCA-3) had the same nucleotide composition as zif268 antisense-ODN but was in an order that has no homology to any sequence in the GenBank database as confirmed by a BLAST search. We also made a $5^{\prime}$-biotinylated nonsense-ODN (5'-GTTGGAGTCGGTGGTTCA-3) for fluorescence immunolabeling.

\section{Fluorescence Immunolabeling}

The presence of zif268 Biotinylated-MSO was detected using the primary antibody (goat anti-biotin IgG; Sigma, St Louis, $\mathrm{MO}$ ), which was recognized using a secondary antibody (rabbit anti-goat IgG-FITC; Sigma). Briefly, brain sections were fixed in $4 \%$ formaldehyde for $1 \mathrm{~h}$, washed twice in PBS, then blocked for $30 \mathrm{~min}$ with blocking solution (5\% BSA, $0.1 \%$ cold water fish skin, $5 \%$ normal serum, all dissolved in PBS). After 1 PBS wash, brain sections were incubated with primary antibody $(1: 800)$ for $16 \mathrm{~h}$ at $4^{\circ} \mathrm{C}$, washed in PBS three times, and then incubated with secondary antibody $(1: 1600)$ for $2 \mathrm{~h}$ at room temperature. Following three final washes in PBS, brain sections were mounted using Vectashield Hard-Set mounting medium (Vector Laboratories, Burlingame, CA) for fluorescence. Each brain section was examined for the antibody FITC under a microscope using a Leica (Nussloch, Germany) I3 filter (450-510 nm) and a mercury light source. Photographs of the right cerebral hemisphere were acquired using a cooled color digital camera (SPOT RT camera; Diagnostic Instruments,
Sterling Heights, MI) and digitized images were stored on a computer diskette.

\section{Statistics}

All analyses were performed with the use of Statview software. One-way ANOVAs were conducted on measures of integrated optical density of the signal generated by in situ hybridization and on behavioral measures of social interaction time. Two ways ANOVA (Sex and Treatment) were conducted when studying the effects of the ASO/MSO treatments in male and female rats. Appropriate post hoc Fisher comparisons were conducted on all ANOVAs to ensure significance. Unpaired $t$-test was used to compare zif268 protein expression in male rats injected with ASO/ MSO oligodeoxynucleotides and zif268 protein expression in $\mathrm{mPFC}$ of male rats $v s$ female rats.

\section{RESULTS}

\section{Sex Differences in the SI Test and the Effects of $17 \beta$-Estradiol (E2) in Females}

In our first experiment, we wanted to know if there were any observable sex differences in social anxiety behaviors as measured by the social interaction test and to examine the role that E2 may be playing in these sex differences. OVX female rats were treated with an acute injection of either OIL or varying doses of $17 \beta$-estradiol $(2,5$, and $10 \mu \mathrm{g})$ and sham surgery male rats were treated with an acute injection of OIL. Forty-eight hours after treatment, all rats were run in the SI test, which consisted of $10 \mathrm{~min}$ of habituation to a novel open field environment, immediately followed by 10 min of social interaction with an unfamiliar intruder rat (intruder females were OVX and males received sham surgery). All rats were killed directly after $20 \mathrm{~min}$ of behavioral testing and brains were collected to be used for zif268 in situ hybridization. Our results revealed a sex difference in the SI test (Figure 1a), where males spent significantly more time engaging in active SI than the OILtreated OVX females $(\mathrm{F}(4,52)=2.11 ; p=0.001)$. In other words, males were essentially less anxious than their female counterparts when tested in the context of environmentdependent social behavior. E2 treatment at each dose tested $(2 \mu \mathrm{g}, 5 \mu \mathrm{g}$, and $10 \mu \mathrm{g})$ did not appear to have an effect on SI in female rats. In addition, there were no differences in locomotor activity during the first $10 \mathrm{~min}$ of exploration of the open field between any of the experimental groups, signifying that males' increased SI was not due to an increase in overall locomotion during testing (Figure 1b).

As E2 did not seem to have an effect on SI in female rats, we examined the hypothesis that females' social interaction during the estrous cycle stages would be identical. For this purpose, we examined social interaction in intact male rats and female rats in either diestrous 1-when levels of E2 are low-or proestrous - when levels of E2 are high - . Our results show that intact male rats (Male, $n=16$ ) show greater social interaction than female rats in both diestrous 1 (FEM D1, $n=16)(\mathrm{F}(2,43)=11.43 ; p=0.0001)$ and in proestrous (FEM P, $n=14)(\mathrm{F}(2,43)=11.43 ; p=0.0003)$. Females in diestrous and proestrous showed similar social interaction (Figure 2). 

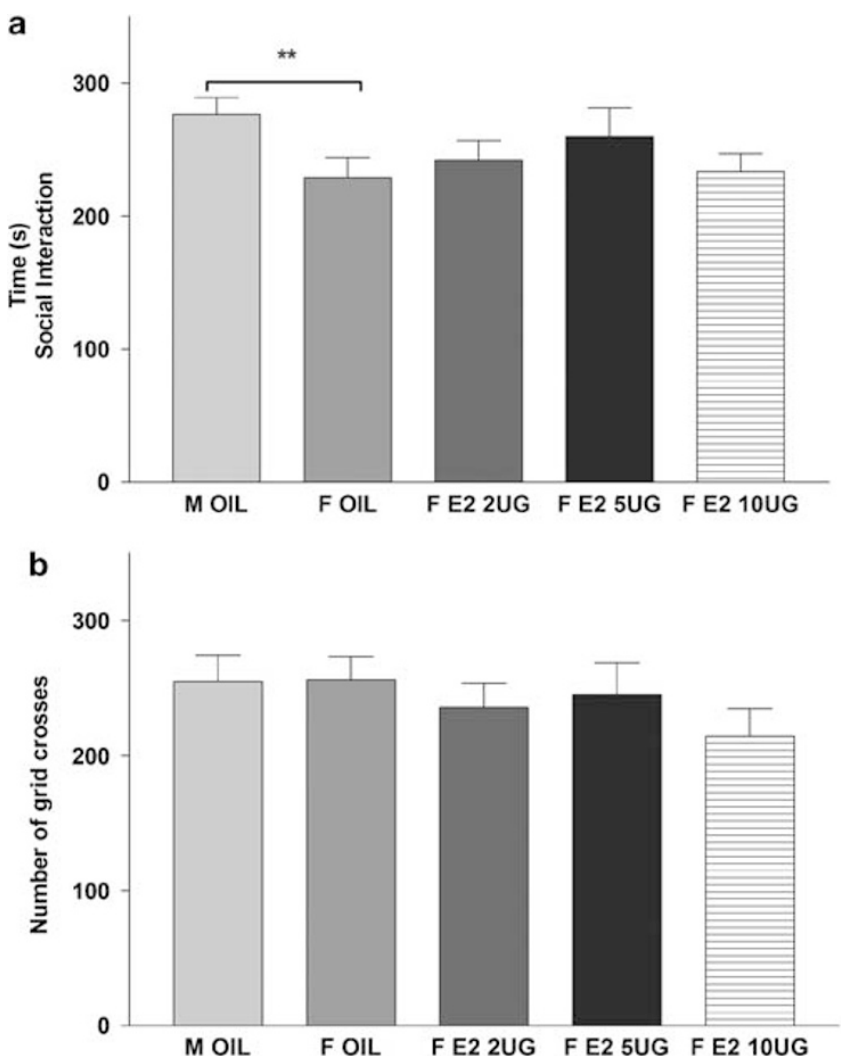

Figure I Sex differences in social interaction behaviors. (a) sham male rats treated with sesame oil ( $M$ OIL, $n=12$ ) spent more time interacting than did female rats treated with sesame oil ( $F$ OIL, $n=12$ ) did. Ovariectomized females treated with $17 \beta$-estradiol at $2 \mu \mathrm{g}$ (F E2 $2 \mathrm{UG}$, $n=12), 5 \mu \mathrm{g}(F$ E2 $5 \cup G, n=12)$ or $10 \mu \mathrm{g}$ (F E2 I0 UG, $n=12$ ) interacted similarly as ovariectomized female rats treated with sesame oil. (b) All experimental groups showed the same locomotor activity during the first $10 \mathrm{~min}$ of exposure to the open field. *Significant difference when compared with M OIL. $* * * 0.01$

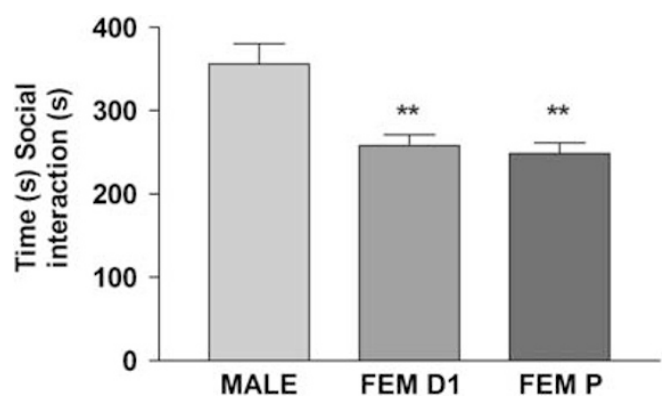

Figure 2 Estrous cycle in female rats does not alter their social interaction: intact male rats (MALE, $n=16$ ) show greater social interaction than female rats in diestrous I (FEM DI, $n=16$ ) or in proestrous (FEM P, $n=14)$. *Significant difference when compared with MALE. *** $p<0.01$.

\section{Sexually Dimorphic zif268 mRNA and Protein Expression in the mPFC}

Following our behavioral study probing sex differences in social anxiety, we wanted to identify key areas of brain activation in males and females killed after exposure to the SI test. To do this, we performed in situ hybridization using a radiolabeled cRNA probe for the immediate-early gene zif268 in sham male rats $(n=8)$ and OVX female rats treated with oil $(n=8)$ and the medium dose of E2 (5 UG $n=8)$. We examined several brain regions involved in emotion, including the dorsal (cingulate cortex 1) and ventral (prelimbic and infralimbic) medial prefrontal cortex (mPFC), dorsal striatum (STR), nucleus accumbens, amygdalar nuclei, and the hippocampal CA1, CA3, and dentate gyrus. In both the dorsal and ventral mPFC, as well as in the STR, we found that males killed following exposure to the SI test had higher zif268 mRNA expression than did OIL and E2-treated OVX females killed under the same conditions (dorsal mPFC: $\mathrm{F}(2,21)=4.89 ; p<0.005$. prelimbic mPFC: $\mathrm{F}(2,21)=4.54 ; p<0.01$. infralimbic mPFC: $\mathrm{F}(2,21)=4.35$; $p<0.05$. striatum $\mathrm{F}(2,21)=4.89 ; p<0.05$ ) (Figures 3 and 4 ). All other brain areas studied showed similar zif268 mRNA expression following the social interaction test (data not shown).

In our next experiment, we asked whether this differential expression of zif268 mRNA in male and female rats was due to the stress of social interaction or whether it is due to an innate genetic difference between male and female rats. To answer this question, we performed another zif268 in situ hybridization comparing basal expression of zif268 mRNA in sham male rats injected with oil $(n=8) v s$ OVX female rats injected with oil $(n=8)$ and killed in basal non-stress conditions $48 \mathrm{~h}$ later. In this experiment, we focused only on MPFC, STR, and CA1 area of the hippocampus. In both the dorsal and ventral $\mathrm{mPFC}$, we found that males killed under basal conditions had higher zif268 mRNA expression than did OVX females killed under the same conditions (dorsal mPFC: $\mathrm{F}(1,14)=8.49 ; p=0.01$ prelimbic mPFC: $\mathrm{F}(1,14)=18.13 ; p=0.0008$ infralimbic mPFC: $\mathrm{F}(1,14)=16.45$; $p=0.002$ ) (Figure 5), indicating that there is sexually dimorphic basal zif268 expression in the mPFC. There were no basal sex differences of zif268 mRNA expression in STR or CA1 (STR: $\mathrm{F}(1,14)=1.71 ; p>0.05$. CA1: $\mathrm{F}(1,14)=0.13$; $p>0.05)$. This sex difference in zif268 expression held true when we examined basal protein expression in intact male rats $(n=7)$ and intact female rats $(n=6)$ in the mPFC $(t=-3.05 ; p<0.0001)$ (Figure 6$)$. Interestingly, these findings suggest a similar pattern between sexually dimorphic zif268 expression in the mPFC and sex differences observed in the SI test, where males had both higher zif268 expression in the mPFC and higher SI than their female counterparts.

\section{zif268 ASO Downregulates zif268 Protein Expression in the mPFC}

In this experiment, we wanted to ascertain that zif268 ASO treatment is effective in downregulating zif268 protein expression in the MPFC. To this end we infused four male rats with zif268 ASO bilaterally in the prelimbic MPFC and four male rats with zif268 MSO bilaterally in the same area. Rats were exposed to the open field and social interaction 70 min later and killed immediately following SI test. Western blot analysis shows that rats treated with zif268 ASO have lower zif268 protein expression in the mPFC (dorsal and ventral) when compared with rats treated with the zif268 MSO $(t=-3.30 ; p=0.01)$. Male rats treated with ASO had about $27 \%$ less zif 268 protein expression in the 

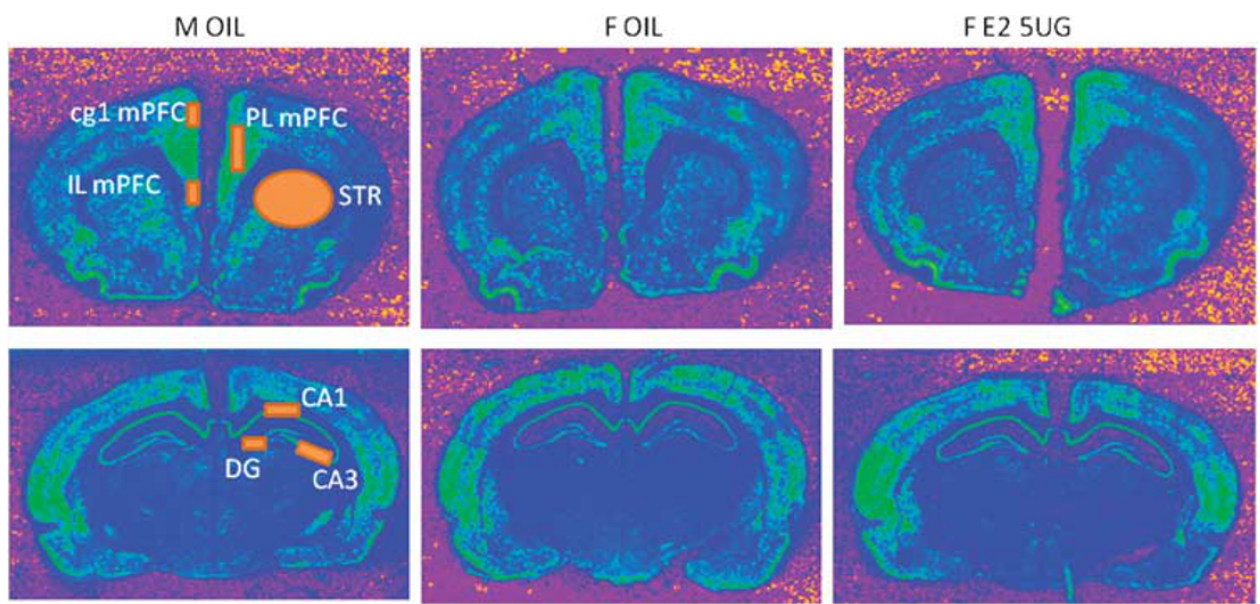

Figure 3 Sex differences in zif268 mRNA expression following social interaction test: Color-enhanced photomicrographs from $x$-ray films exposed for 5 days after in situ hybridization with antisense cRNA probe against zif268 mRNA. These representative images show that sham male rats treated with oil ( $\mathrm{M}$ OIL) have greater zif268 expression than ovariectomized female rats treated with oil (F OIL) or with E2 $5 \cup G$ (F E2 $5 \cup G)$ in the cingulate cortex I (cg I mPFC), prelimbic (PL mPFC), infralimbic (IL mPFC) and striatum (STR). M OIL, F OIL and F E2 $5 \cup G$ show, however, similar mRNA expression in the hippocampal areas: CAI, CA3 and DG. The orange outlines show an example of where zif268 mRNA was sampled for quantification.

mPFC when compared with rats treated with zif268 MSO (Figure 7).

\section{Downregulation of zif268 in the mPFC in Male Rats Abolishes Sex Differences in the Social Interaction Test but does not Affect Open Field Measures}

Based on the previous findings, we next sought to determine if zif268 had a functional role in mediating sex differences found in social anxiety-like behaviors in the SI test. In other words, we wanted to know if a decrease in zif268 expression resulting from infusion of a zif268 antisense oligodeoxynucleotide (zif268 ASO) into the prelimbic mPFC in male rats would decrease their SI and make them more similar to females in terms of social anxiety behavior measured by the SI test. We wanted also to ascertain whether or not zif268 has a role in social interaction in female rats. To this aim, female rats were also treated with zif268 ASO and their SI was compared with female rats treated with zif268 MSO. Because we found no effects of E2 or any estrous cycle effects on SI behavior (Figure 2), we used intact female rats for this experiment. In this study, both male and female rats underwent stereotaxic surgery with bilateral cannulae aimed at the prelimbic mPFC. To ensure proper infusion of zif268 within the MPFC, a biotinylated-zif268 missense oligodeoxynucleotides (zif268 B-MSO) were infused into the mPFC of four male rats before the commencement of the experiment. Their brains were collected, sectioned and used for fluorescence immunolabeling against biotin. The results ensured us that the zif268 construct was diffusing within the prelimbic area and cingulate cortex 1 of the mPFC (Figure 8d). Males and females were given infusions of zif268 MSO or zif268 ASO in the prelimbic mPFC, and $70 \mathrm{~min}$ later all rats were tested in the open field for $10 \mathrm{~min}$ followed by social anxiety-like behavior in the SI test as described above. During the first $10 \mathrm{~min}$ in the open field, all rats showed similar locomotor activity $(\mathrm{F}(1,30)=0.03$; $p=0.86$ ) (Figure 8a) and similar time in the center of the open field $(\mathrm{F}(1,30)=0.43 ; p=0.51)$ (Figure $8 \mathrm{~b})$. During the social interaction test, two way ANOVA revealed no sex effect $(\mathrm{F}(1,30)=0.18 ; p=0.67)$, but a significant treatment effect $(\mathrm{F}(1,30)=4.81 ; p=0.03)$ and a significant interaction between sex and treatment $(\mathrm{F}(1,30)=9.06 ; p=0.005)$. Further post hoc analysis show that male rats treated with zif268 MSO show greater SI than male rats treated with the zif268 ASO construct $(p=0.0008)$ and greater SI than female rats treated with zif268 MSO $(p=0.01)$. Female rats treated with zif268 MSO or zif268 ASO showed the same SI $(p=0.57)$ (Figure $8 c)$.

\section{DISCUSSION}

In this study, we set out to determine which factors were important in mediating sex differences in the SI test, an anxiety test with a strong social component. In our first experiment, we found that acute $17 \beta$-estradiol (E2) treatment in OVX females had no effects on social anxiety in the SI test. We have also found that estrous cycle stages do not affect social interaction in intact female rats. Interestingly, we found zif268 expression, however, to be sexually dimorphic in the dorsal and ventral mPFC in both basal conditions and after exposure to the SI test, with males having higher zif268 expression than females. This was similar with sex differences found in measures of social anxiety-like behavior in the SI test, where males had higher SI than did females, signifying that males were less socially anxious than females in this paradigm. Once zif268 expression was downregulated bilaterally in the mPFC of male rats, sex differences in SI were abolished and the males showed decreased SI comparable with that of female SI behavior. Essentially, a decrease in zif268 expression in the mPFC of male rats was anxiogenic and made them behave just as anxious as females during the SI test. This effect was specific to social anxiety as the same zif268 ASO/MSO treatments did not affect anxiety-like behaviors in the open field test. Interestingly, the downregualtion of zif268 in female rats was without consequence on their social 

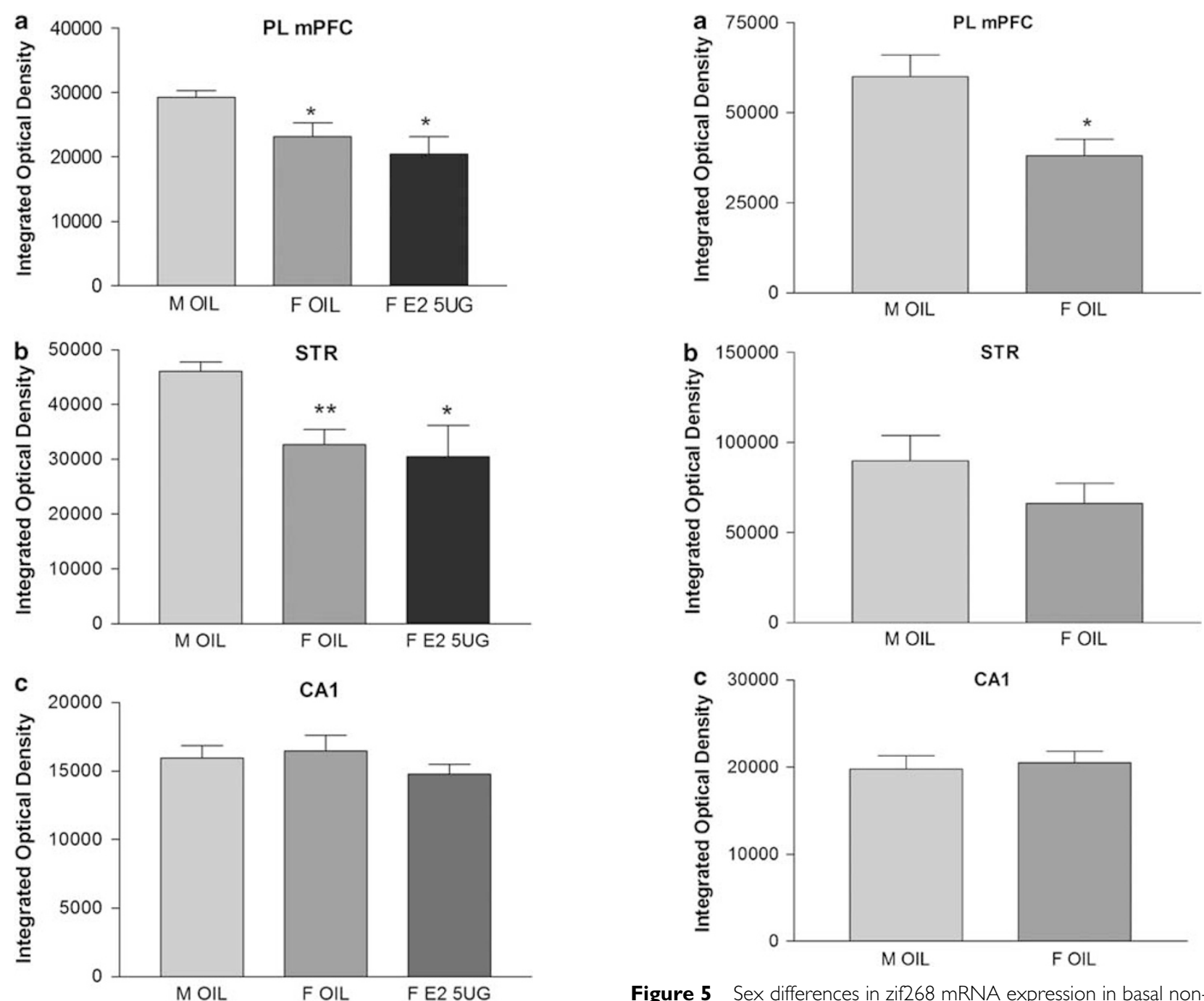

CA1

Figure 4 Sex differences in zif268 mRNA expression following social interaction test: sham male rats $(M O I L, n=8)$ showed greater zif268 mRNA expression in the $\mathrm{cgl}, \mathrm{PL}$ and IL medial prefrontal cortex (only PL mPFC is represented in (a)) and dorsal striatum (STR) (b) when compared with ovariectomized female rats treated with oil ( $F$ OIL, $n=8$ ) or treated with E2 $5 \cup G(n=8)$. The three groups showed the same zif268 mRNA expression in hippocampal CAI region, CA3, and DG (only CAI is represented in (c)). ${ }^{*} p<0.05,{ }^{*} * 0<0.01$.

interaction behaviors. Thus, we were able to implicate the immediate-early gene zif 268 in the $\mathrm{mPFC}$ as having a functional role in mediating sex differences in social anxiety in the SI test. Our findings show for the first time that zif268 expression in the mPFC has a profound effect on sex differences in social anxiety-like behavior in rats.

\section{The Effects of $17 \beta$-Estradiol and Stages of Estrous Cycle on Female Social Anxiety}

One of our main results was that, while there were appreciable sex differences in social interaction behaviors, acute E2 replacement had no effect on social interaction behaviors of OVX females, and various stages of estrous cycle did not affect social interaction. Our findings add to the existing body of literature on the role of $17 \beta$-estradiol

(E2) on anxiety in females, which is seemingly contradictory at best. Human studies have found that E2 treatment in postmenopausal women given E2 treatment had either positive effects (Best et al, 1992; Miller et al, 2002), no effects (Cummings and Brizendine, 2002; Schleifer et al, 2002), or dose-dependent negative effects on mood (Bjorn et al, 2003; Newhouse et al, 2008). Similarly, several studies in rats have found that acute E2 treatment has no effects on anxiety-like behavior (Martinez-Mota et al, 2000; Nomikos and Spyraki, 1988). Short-term (or subchronic) E2 treatment, lasting from 3 to 7 days, has been shown to have both anxiolytic-like effects (Koss et al, 2004; McCarthy, 1995; Nomikos and Spyraki, 1988) and anxiogenic-like effects (Koss et al, 2004; Morgan and Pfaff, 2002) in female rats, depending sometimes on which tests were used to measure anxiety-like behavior.

A plausible explanation for our findings that E2 or estrous cycle has no effect on SI behavior stems from the fact that 

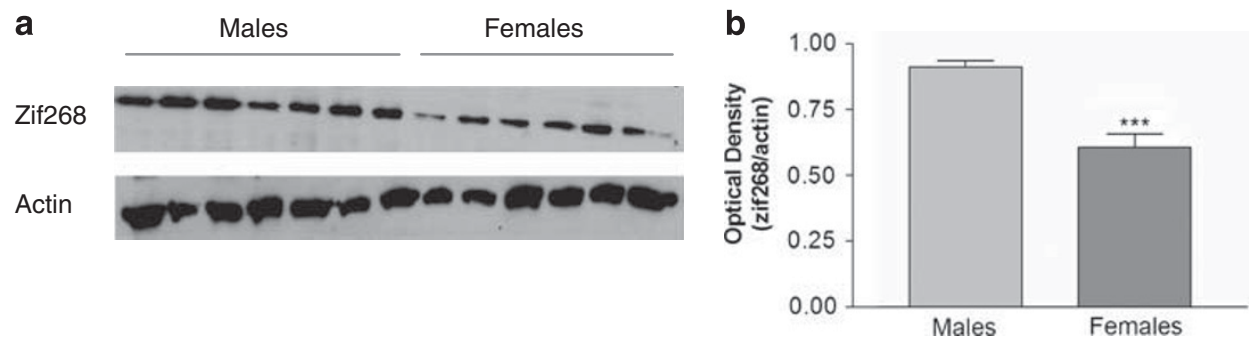

Figure 6 Sex differences in zif268 protein in mPFC in basal non-stress conditions: intact male rats $(n=7)$ show greater zif268 protein expression than intact female rats $(n=6)$ in mPFC. (a) Western blots from mPFC lysate show that male rats have more zif268 protein expression than female rats. (b) Western blot quantified data are shown as optical density of zif268 expression divided by optical density of actin for each rat. **** $<0.001$.
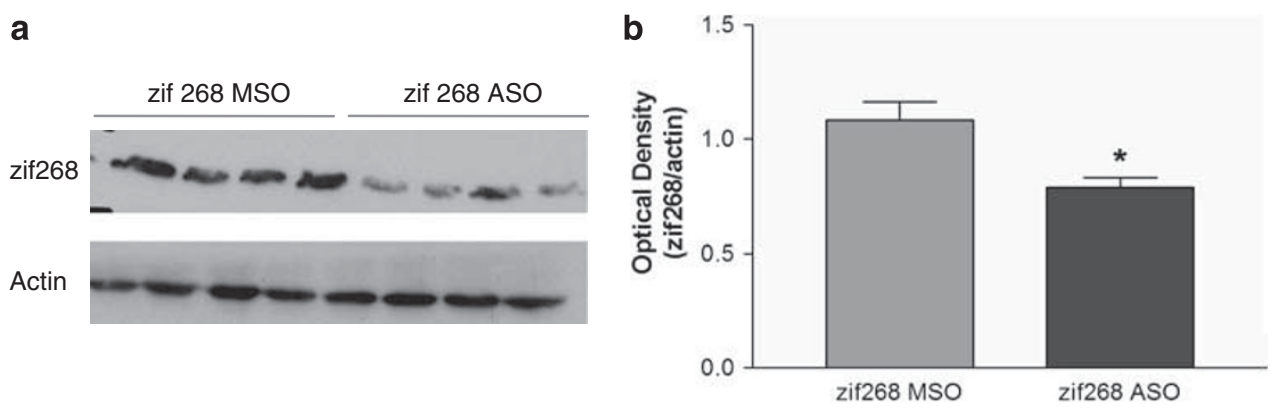

Figure 7 zif268 ASO reduces zif268 protein expression in the mPFC: intact male rats treated with zif268 ASO ( $n=4)$ show lower zif268 protein expression than male rats treated with zif268 MSO $(n=4)$. (a) Western blots from mPFC lysate show that rats treated with zif268 MSO have more zif268 protein expression than male rats treated with zif268 ASO. Zif268 MSO and zif268 ASO-treated rats have similar actin protein expression. (b) Western blot quantified data are shown as optical density of zif268 expression divided by optical density of actin for each rat. *p $<0.05$.

E2 is known to enhance sexual receptivity and sexual motivation to seek out a mate, shown by studies in which E2-treated OVX females preferred interaction with a male conspecific over a female in partner preference tests (Edwards and Pfeifle, 1983). These accounts could help to explain why acute E2 treatment administered to OVX females failed to produce any observable effects on social anxiety as measured by same-sex SI behavior. Perhaps it is the case that the E2 treatment served to offset the female rats' innate motivation for SI through social avoidance of same-sex conspecifics, thereby resulting in no net effect of E2 treatment in OVX females or when comparing diestrous to proestrous females in measures of SI.

One of the limitations of this work is that only one aspect of anxiety-like behaviors was studied. If other measures of anxiety-like behaviors have been included in the study (ie, Elevated plus maze, Elevated zero maze, Black and white box, Holeboard test, Aron test, Vogel test, and Geller Seifter test) we would have had a definitive answer as to whether E2 and estrous cycle stages affect generalized anxiety-like behaviors in rodents. It is possible that $\mathrm{E} 2$ or the various estrous stages might not have any effect on social anxiety in females but they may alter other aspects of anxiety-like behaviors (ie, fear of heights, fear of open spaces, and fear of light...etc).

\section{The Role of mPFC in Anxiety}

Abnormal functioning of the mPFC has been associated with many psychological disorders in humans, including schizophrenia, obsessive-compulsive disorder, and depression (Moghaddam and Homayoun, 2008). Its role in anxiety, however, is still unclear. Studies examining the effects of lesions in the mPFC on anxiety-like behavior in rodents have yielded seemingly contradictory results. Some studies have found that $\mathrm{mPFC}$ lesions have an anxiolytic effect in the elevated plus maze, shock-probe burying test, and the SI test (Gonzalez et al, 2000; Lacroix et al, 2000). Other studies have indicated anxiogenic effects after either mPFC lesions or infusions of FG7142, a benzodiazepine inverse agonist, into the mPFC. These findings include decreased time spent in the center of an open field and decreased time spent in the open arms of the elevated plus maze (Jaskiw and Weinberger, 1990; Morgan et al, 1993). Although these studies used a range of techniques to either lesion or chemically inhibit mPFC function, our study is the first that we know of to use temporary and reversible downregulation of an inducible transcription factor in the dorsal and ventral mPFC to study its effects on social interaction behavior.

In addition to the mPFC being implicated in anxiety and other mental disorders, it has also been widely investigated in terms of social behavior and social cognition. Clinical observations have found that patients with damage to the prefrontal cortex, especially the orbitofrontal cortex (OFC) and the mPFC, commonly display socially inappropriate behavior (Anderson et al, 1999). Damage specifically to the mPFC results in mental and behavioral deficits including social inappropriateness, lack of judgment, and inappropriate affect (Barrash et al, 2000), as well as impairments in social decision making (Bechara et al, 1999). Activation of the $\mathrm{mPFC}$ is observed during subjects' perception of social interaction through facial expressions and during their experiences of personal 

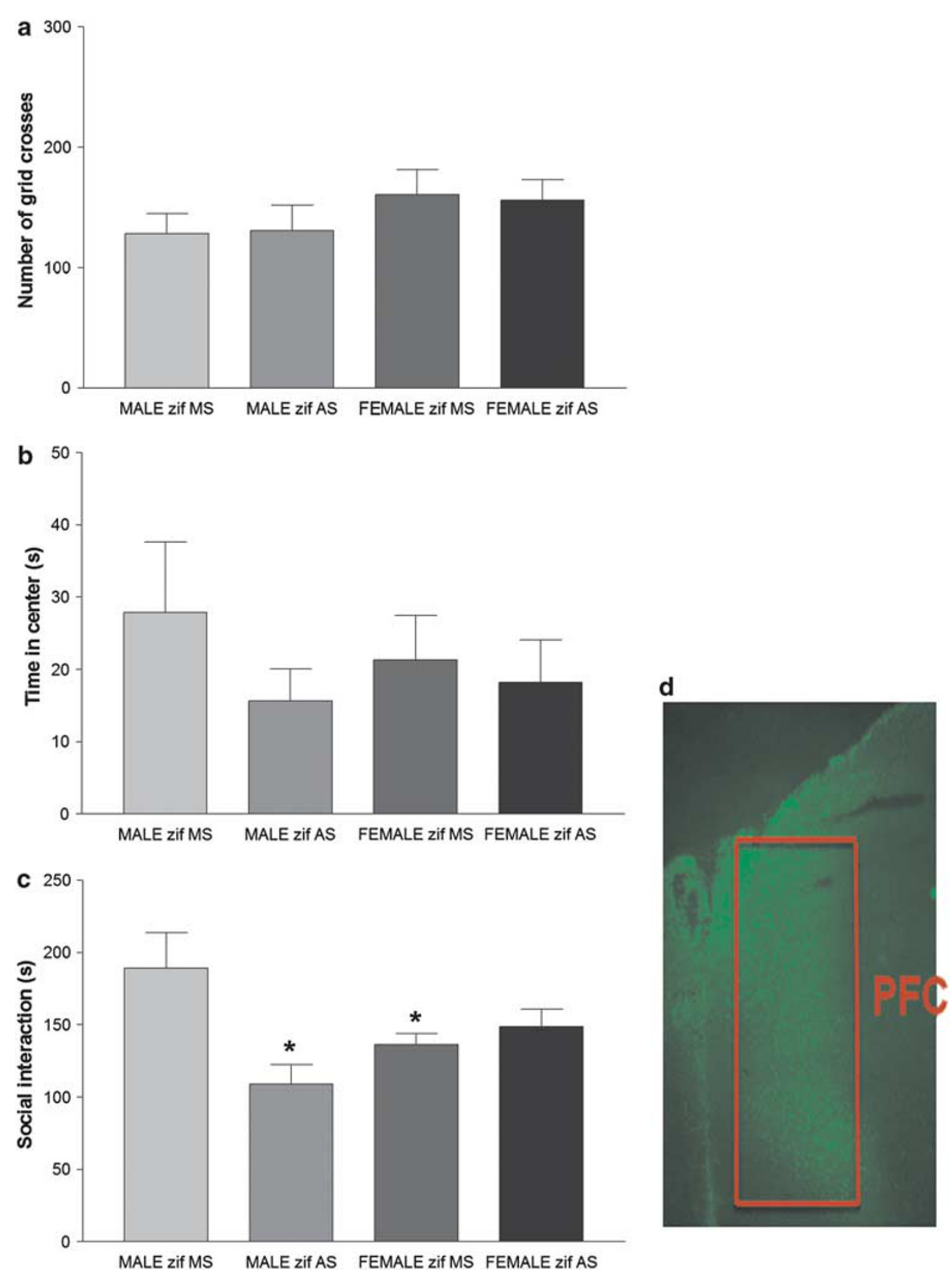

Figure 8 Downregulation of zif268 in male rats, but not in female rats, abolishes sex differences in social interaction: (a) intact male and female rats treated zif268 missense or antisense oligodeoxynucleotide exhibit similar locomotor activity during the open field test. (b) intact male and female rats treated zif268 missense or antisense oligodeoxynucleotide spend similar time in the center of the open field. (c) intact male rats treated with zif268 missenseoligodeoxynucleotide (MALE zif MS, $n=8$ ) show greater social interaction than female rats treated with the same construct (FEMALE zif MS, $n=10$ ). This sex difference in social interaction is abolished when male rats are treated with zif268 antisense (MALE zif AS, $n=9$ ). Female rats treated with zif268 MSO or zif268 AS (FEMALE zif AS, $n=7$ ) do not differ in their social interaction time. *Significant difference when compared with MALE zif MS. (d) extent of diffusion of biotinylated-zif268-MSO within the mPFC. The diffusion covered the cingulate cortex I and prelimbic mPFC. $* p<0.05$.

involvement through eye gaze (Schilbach et al, 2006). Given this evidence, it is clear that the mPFC is important in regulating social behavior in humans and studies regarding the $\mathrm{mPFC}$ and animal social behavior have yielded corroborating evidence. In primates, lesions to the mPFC result in abnormal social behavior: monkeys with mPFC lesions are socially isolated, withdrawn, and less apt to explore their environment (Myers et al, 1973). In two recent studies, male rats with lesions or inhibitions to different areas of the frontal cortex - cingulate cortex and ventral mPFC - displayed impaired social interaction, social memory, and reduced anxiety (Resstel et al, 2008; Rudebeck et al, 2007). As our zif268 ASO/MSO constructs diffused to the prelimbic and cingulate cortex 1 we cannot ascertain that zif268 in dorsal mPFC or zif268 in ventral mPFC has a more important role in social anxiety. Future studies will be needed to dissect the role of these two areas in social anxiety. 
Given the nature of the anxiety test that we employed in our study, we must consider the SI results as not only indicative of an animal's anxiety level, but also as a measure of its social behavior in general. In this respect, our study established that temporary downregulation of zif268 expression in males caused a marked decrease in their sex-specific social interaction and leads to males displaying a female-like phenotype in terms of social behavior.

\section{Zif268 and its Role in Behavior}

Zif268 has previously been implicated in several complex behaviors, such as learning and memory formation (Cole et al, 1989), fear conditioning (Ressler et al, 2002); (Malkani et al, 2004), and drug addiction (Valjent $e t$ al, 2006). In one study, investigating anxiety-like behavior through the use of the fear-conditioning paradigm, zif268 antisense oligodeoxynucleotides (ASO) infused into the amygdala of male rats abolished freezing (and hence, fear conditioned) behaviors in those animals (Malkani et al, 2004). Despite this relatively large body of evidence establishing a role for zif268 in certain behaviors with a strong learning and memory component, to the best of our knowledge there have been no investigations into whether zif268 expression in the brain varies between the sexes and how sex differences in zif268 expression patterns in various brain regions might influence complex behavior. Here, we present that there are indeed site-specific differences in the levels of zif268 mRNA and protein expression when examining the $\mathrm{MPFC}$ in males $v s$ females, with males showing higher zif268 mRNA and protein expression than females. This sexual dimorphism in zif268 expression in the mPFC was also found to be similar to the pattern of sex differences in social anxiety-like behavior as measured by the SI test, where males had both higher levels of zif268 mRNA expression in the mPFC and lower social anxiety than females. Moreover, when zif268 expression is temporarily downregulated in the mPFC of male rats, their levels of social anxiety-like behavior are significantly increased and their behavioral phenotype becomes similar to that of female rats tested in the same social anxiety paradigm. However, changes in zif268 in mPFC do not seem to affect females' SI behaviors. Interestingly, these effects were specific to the social anxiety-like behaviors as open field measures were not affected by zif268 ASO/MSO treatments.

It is also important to note that zif268 mRNA expression showed differential regulation in the dorsal striatum following social interaction. Indeed, male rats showed greater zif268 expression than female rats following the social interaction test. In light of the data showing that zif268 expression in the striatum has a role in memory and motivation (Bozon et al, 2003; Fumagalli et al, 2009), it is possible that the high zif268 expression in the striatum of male rats promotes greater social interaction. It is reasonable to suggest that zif268 within the corticostriatal circuitry may have a major role in social interaction, a possibility that is worth further investigations.

In our experiment, the effects of zif268 ASO on social interaction in male rats were observed within $90 \mathrm{~min}$ of its infusion. This time is enough for decreased zif268 protein to affect transcription and translation of some critical genes that have a role in social interaction. But, we should keep in mind that zif268 has a $\mathrm{C} 2 \mathrm{H} 2$ zinc finger domain that allows it to interact with other proteins (Brayer and Segal, 2008), and it is this interaction of zif268 with other proteins that may be important in determining social interaction. It is also possible that zif268 can affect social interaction through both genomic and non-genomic effects (proteinprotein interactions).

It is not clear why zif268 in mPFC is implicated in the social interaction of male rats but not female rats. But one may hypothesize that there might be a need for a certain high continuous threshold of zif 268 expression in $\mathrm{mPFC}$ to maintain high social interaction. This threshold of zif268 could be maintained by testosterone in male rats. One could also hypothesize that the downstream gene targets of zif268 are different in male and female rats (genomic effect) or that zif268 is interacting with specific proteins found only in male rats - may be genes associated with the $\mathrm{Y}$ chromosome-, this interaction may be critical for enhancing males' SI behaviors (non-genomic effect). These hypotheses need to be further investigated.

In conclusion, our study revealed that sexually dimorphic expression patterns of a key molecular factor in the mPFC, the immediate-early gene zif268, can serve to mediate sex differences in anxiety-like behavior having a strong social component. Finally, much remains to be known as far as how and why zif268 expression in the MPFC contributes to sex differences in SI behavior; in fact, we believe that our study raises many more important questions that we will aim to answer in the future. Indeed it is of great importance that we continue to delve into the neurobiological mechanisms underlying sex differences in behavior as well as in psychopathologies such as social anxiety and mood disorders and that we persist in advancing the boundaries in this newly emerging research area.

\section{DISCLOSURE}

The author(s) declare that, except for income received from my primary employer and NIH (Grant 5R03DA021554-02), no financial support or compensation has been received from any individual or corporate entity over the past 3 years for research or professional service and there are no personal financial holdings that could be perceived as constituting a potential conflict of interest.

\section{REFERENCES}

Anderson SW, Bechara A, Damasio H, Tranel D, Damasio AR (1999). Impairment of social and moral behavior related to early damage in human prefrontal cortex. Nat Neurosci 2: 1032-1037.

Barrash J, Tranel D, Anderson SW (2000). Acquired personality disturbances associated with bilateral damage to the ventromedial prefrontal region. Dev Neuropsychol 18: 355-381.

Bechara A, Damasio H, Damasio AR, Lee GP (1999). Different contributions of the human amygdala and ventromedial prefrontal cortex to decision-making. J Neurosci 19: 5473-5481.

Best NR, Rees MP, Barlow DH, Cowen PJ (1992). Effect of estradiol implant on noradrenergic function and mood in menopausal subjects. Psychoneuroendocrinology 17: 87-93.

Bjorn I, Sundstrom-Poromaa I, Bixo M, Nyberg S, Backstrom G, Backstrom T (2003). Increase of estrogen dose deteriorates mood during progestin phase in sequential hormonal therapy. J Clin Endocrinol Metab 88: 2026-2030. 
Bozon B, Davis S, Laroche S (2003). A requirement for the immediate early gene zif268 in reconsolidation of recognition memory after retrieval. Neuron 40: 695-701.

Brayer KJ, Segal DJ (2008). Keep your fingers off my DNA: proteinprotein interactions mediated by $\mathrm{C} 2 \mathrm{H} 2$ zinc finger domains. Cell Biochem Biophys 50: 111-131.

Cole AJ, Saffen DW, Baraban JM, Worley PF (1989). Rapid increase of an immediate early gene messenger RNA in hippocampal neurons by synaptic NMDA receptor activation. Nature 340: 474-476.

Cummings JA, Brizendine L (2002). Comparison of physical and emotional side effects of progesterone or medroxyprogesterone in early postmenopausal women. Menopause 9: 253-263.

Davis HP, Squire LR (1984). Protein synthesis and memory: a review. Psychol Bull 96: 518-559.

Edwards DA, Pfeifle JK (1983). Hormonal control of receptivity, proceptivity and sexual motivation. Physiol Behav 30: 437-443.

File SE, Seth P (2003). A review of 25 years of the social interaction test. Eur J Pharmacol 463: 35-53.

Fumagalli F, Franchi C, Caffino L, Racagni G, Riva MA, Cervo L (2009). Single session of cocaine intravenous self-administration shapes goal-oriented behaviours and up-regulates Arc mRNA levels in rat medial prefrontal cortex. Int J Neuropsychopharmacol 12: 423-429.

Gater R, Tansella M, Korten A, Tiemens BG, Mavreas VG, Olatawura MO (1998). Sex differences in the prevalence and detection of depressive and anxiety disorders in general health care settings: report from the World Health Organization Collaborative Study on Psychological Problems in General Health Care. Arch Gen Psychiatry 55: 405-413.

Goelet P, Castellucci VF, Schacher S, Kandel ER (1986). The long and the short of long-term memory-a molecular framework. Nature 322: 419-422.

Gonzalez LE, Rujano M, Tucci S, Paredes D, Silva E, Alba G et al (2000). Medial prefrontal transection enhances social interaction. I: behavioral studies. Brain Res 887: 7-15.

Hebb MO, Robertson HA (1997). Coordinate suppression of striatal ngfi-a and c-fos produces locomotor asymmetry and upregulation of IEGs in the globus pallidus. Brain Res Mol Brain Res 48: 97-106.

Jaskiw GE, Weinberger DR (1990). Ibotenic acid lesions of the medial prefrontal cortex potentiate FG-7142-induced attenuation of exploratory activity in the rat. Pharmacol Biochem Behav 36: 695-697.

Johnston AL, File SE (1991). Sex differences in animal tests of anxiety. Physiol Behav 49: 245-250.

Kabbaj M, Devine DP, Savage VR, Akil H (2000). Neurobiological correlates of individual differences in novelty-seeking behavior in the rat: differential expression of stress-related molecules. J Neurosci 20: 6983-6988.

Kessler RC, McGonagle KA, Zhao S, Nelson CB, Hughes M, Eshleman S et al (1994). Lifetime and 12-month prevalence of DSM-III-R psychiatric disorders in the United States. Results from the National Comorbidity Survey. Arch Gen Psychiatry 51: 8-19.

Koss WA, Gehlert DR, Shekhar A (2004). Different effects of subchronic doses of 17-beta estradiol in two ethologically based models of anxiety utilizing female rats. Horm Behav 46: 158-164.

Lacroix L, Spinelli S, Heidbreder CA, Feldon J (2000). Differential role of the medial and lateral prefrontal cortices in fear and anxiety. Behav Neurosci 114: 1119-1130.
Lisman JE (1999). Relating hippocampal circuitry to function: recall of memory sequences by reciprocal dentate-CA3 interactions. Neuron 22: 233-242.

Malkani S, Wallace KJ, Donley MP, Rosen JB (2004). An egr-1 (zif268) antisense oligodeoxynucleotide infused into the amygdala disrupts fear conditioning. Learn Mem 11: 617-624.

Martinez-Mota L, Estrada-Camarena E, Lopez-Rubalcava C, Contreras CM, Fernandez-Guasti A (2000). Interaction of desipramine with steroid hormones on experimental anxiety. Psychoneuroendocrinology 25: 109-120.

McCarthy MM (1995). Estrogen modulation of oxytocin and its relation to behavior. Adv Exp Med Biol 395: 235-245.

Miller KJ, Conney JC, Rasgon NL, Fairbanks LA, Small GW (2002). Mood symptoms and cognitive performance in women estrogen users and nonusers and men. J Am Geriatr Soc 50: 1826-1830.

Moghaddam B, Homayoun H (2008). Divergent plasticity of prefrontal cortex networks. Neuropsychopharmacology 33: 42-55.

Morgan MA, Pfaff DW (2002). Estrogen's effects on activity, anxiety, and fear in two mouse strains. Behav Brain Res 132: 85-93.

Morgan MA, Romanski LM, LeDoux JE (1993). Extinction of emotional learning: contribution of medial prefrontal cortex. Neurosci Lett 163: 109-113.

Myers RE, Swett C, Miller M (1973). Loss of social group affinity following prefrontal lesions in free-ranging macaques. Brain Res 64: 257-269.

Newhouse PA, Dumas J, Hancur-Bucci C, Naylor M, Sites CK, Benkelfat C et al (2008). Estrogen administration negatively alters mood following monoaminergic depletion and psychosocial stress in postmenopausal women. Neuropsychopharmacology 33: 1514-1527.

Nomikos GG, Spyraki C (1988). Influence of oestrogen on spontaneous and diazepam-induced exploration of rats in an elevated plus maze. Neuropharmacology 27: 691-696.

Ressler KJ, Paschall G, Zhou XL, Davis M (2002). Regulation of synaptic plasticity genes during consolidation of fear conditioning. J Neurosci 22: 7892-7902.

Resstel LB, Souza RF, Guimaraes FS (2008). Anxiolytic-like effects induced by medial prefrontal cortex inhibition in rats submitted to the Vogel conflict test. Physiol Behav 93: 200-205.

Rudebeck PH, Walton ME, Millette BH, Shirley E, Rushworth MF, Bannerman DM (2007). Distinct contributions of frontal areas to emotion and social behaviour in the rat. Eur J Neurosci 26: $2315-2326$.

Schilbach L, Wohlschlaeger AM, Kraemer NC, Newen A, Shah NJ, Fink GR et al (2006). Being with virtual others: neural correlates of social interaction. Neuropsychologia 44: 718-730.

Schleifer LA, Justice AJ, de Wit H (2002). Lack of effects of acute estradiol on mood in postmenopausal women. Pharmacol Biochem Behav 71: 71-77.

Schneier FR, Johnson J, Hornig CD, Liebowitz MR, Weissman MM (1992). Social phobia. Comorbidity and morbidity in an epidemiologic sample. Arch Gen Psychiatry 49: 282-288.

Valjent E, Aubier B, Corbille AG, Brami-Cherrier K, Caboche J, Topilko P et al (2006). Plasticity-associated gene Krox24/Zif268 is required for long-lasting behavioral effects of cocaine. J Neurosci 26: 4956-4960.

Walf AA, Frye CA (2005). Antianxiety and antidepressive behavior produced by physiological estradiol regimen may be modulated by hypothalamic-pituitary-adrenal axis activity. Neuropsychopharmacology 30: 1288-1301. 